\title{
Right Atrial Thrombus Mimicking Myxoma with Subclinical Pulmonary Embolism
}

\author{
Artemiou P, Lukacin S, Bily B \\ University of P. J. Safarik, Medical Faculty, Dept. of Cardiovascular Surgery, VUSCH a. s., \\ Kosice, Slovakia
}

Key words:

Right atrial thrombus,

Pulmonary embolism, Myxoma.

\section{Abstract:}

We reported a case of right atrial thrombosis mimicking myxoma in a patient with atrial fibrillation and subclinical pulmonary embolism. The definite diagnosis was made postoperatively. While sometimes the diagnosis is challenging, these patients are at high risk for pulmonary embolism and they need prompt intervention.

\section{Introduction:}

Non-valvular atrial fibrillation is a common problem in the elderly, occurring in $2 \%$ to $4 \%$ of the population $>60$ years of age and it is one of the most common causes of embolic systemic events.

We report a case of intracardiac thrombosis mimicking myxoma as a complication of atrial fibrillation associated with a prothrombotic state.

\section{Case Report:}

A 60 year old patient was presented with a right atrial mass and a 3 vessel coronary artery disease. The patient had been sufferring from persistent atrial fibrillation. In the past he had a transitory ischemic attack and a lower leg embolism and also he did not receive any anticoagulation therapy.

He underwent coronary angiography which showed 3 vessels coronary artery disease. Transthoracic echocardiogram showed a freefloating right atrial mass of size $7.0 \times 1.1 \mathrm{~cm}$ with a highly embolic potential suggesting myxoma or thrombus and is shown in figure 1.

Patient underwent an emergency surgery. After institution of the cardiopulmonary bypass he underwent surgical exploration of the right heart chambers and pulmonary arteries with pulmonary embolectomy. No thrombi were found in the right atrium and ventricle but were found in the main, right and left pulmonary artery. $\mathrm{He}$ underwent a left internal thoracic artery by-pass
(Cardiovasc. j. 2013; 6(1): 71-73)

graft to left anterior descending artery and a great saphenous vein by-pass graft to the marginal branch.

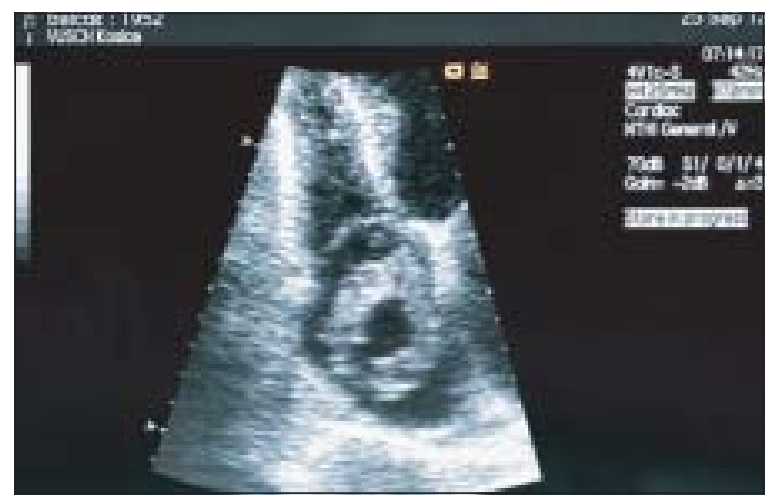

Fig.-1: Transthoracic echocardiography, apical two chambers view showing the right atrial mass during systole.

The postoperative course was uneventful. The pulmonary embolism was subclinical. Gross examination of the operative specimen showed pieces of fresh thrombi which were confirmed later also by a histologic examination, and are shown in figure 2.

A search for a hypercoagulable state revealed normal thrombin, prothrombin and partial thromboplastin times, normal antitrhombin III, protein $\mathrm{C}$ and $\mathrm{S}$ and homocystein levels. Screening for anticardiolipin antibodies and lupus anticoagulant were negative. Genetic analysis showed heterozygous mutations of FXII

Address of Correspondence: Panagiotis ARTEMIOU, MD PhD. University of P. J. Safarik, Medical Faculty, Dept. of Cardiovascular Surgery, VUSCH a. s., Kosice, Slovakia. e-mail: panayiotisartemiou@yahoo.com 


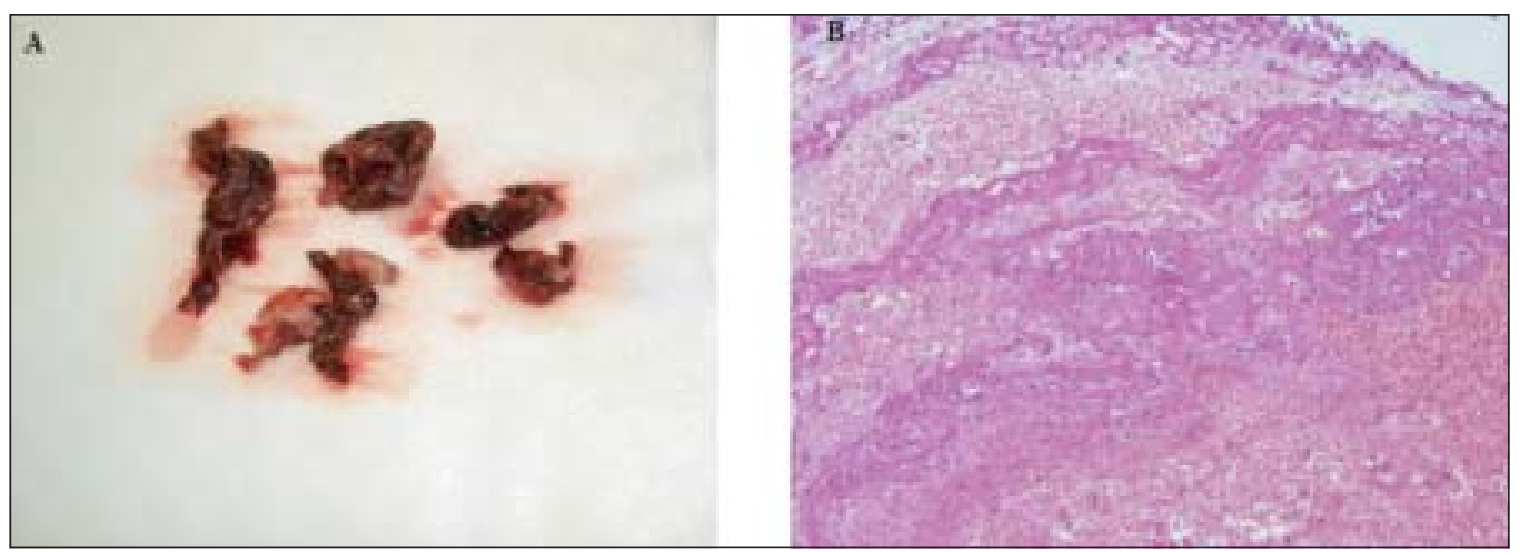

Fig.-2. A) Operative specimen showing pieces of thrombi after the pulmonary embolectomy. B) Histologic examination reveals fresh thrombus. It shows alternate layers of fibrin-platelet material and red cells.

C46T and PAI-1 (plasminogen activator inhibition -1). D-dimer and fibrinogen levels were increased showing an activated systemic prothrombotic and fibrinolytic state.

The patient received antithrombotic treatment with a low molecular heparin and warfarin and on postoperative day 7 he was discharged from the hospital in good and stable condition.

\section{Discussion:}

In atrial fibrillation the fibrillating left atrium creates low-flow conditions. In addition various hypercoagulable state markers such as factor VIII, fibrinogen, D-dimer, and von Willebrand factor have been shown to increase as in our patient who had an increased D-dimer levels., ${ }^{1,2}$

Right atrial and also left atrial thrombi can develop in several situations. First, hyperhomocysteinemia is associated with the presence of left atrial thrombus in stroke patients with non-valvular atrial fibrillation where homocysteine alters the thrombotic properties of the endothelium. ${ }^{3}$

Moreover, intracardiac thrombosis associated with pulmonary embolism is also reported in patients with cardiac amyloidosis ${ }^{4}$ and in patients with hypereosinophilic syndrome. ${ }^{5}$ Also the risk of intracardiac thrombosis is increased in patients who have systemic lupus erytematosus with positive lupus anticoagulant activity or have medium or high levels of anticardiolipin antibodies. ${ }^{6}$
Finally, intracardiac thrombosis with pulmonary embolism is described in Bechet's disease and in heparin-induced thrombocytopenia. ${ }^{7,8}$

The differential diagnosis of right atrial mass includes vegetation, tumor and thrombus. ${ }^{9,10}$ The most common primary tumor is the myxoma and fifteen percent of them arise in the right atrium and clinically both myxoma and thrombi can present with a pulmonary embolism. From the patient's history or examination there was nothing to suggest infective bacterial endocarditis or malignancy. In our patient preoperative investigations could not differentiate such a thrombus from a myxoma and the diagnosis was made postoperatively.

In conclusion it remains unknown whether prolonged heparin administration, thrombolysis, high-intensity anticoagulation with warfarin or surgical exploration is the best therapeutic approach. Regardless, the great size of the mass and the mobile appearance on echocardiography seemed to place our patient at high risk for pulmonary embolism and led us to choose the surgical exploration and the coronary artery bypass grafting.

\section{Conclusion:}

We reported a case of right atrial thrombosis mimicking myxoma in a patient with atrial fibrillation and subclinical pulmonary embolism. The definite diagnosis was made postoperatively. While sometimes the diagnosis is challenging, these patients are at high risk for pulmonary embolism and they need prompt intervention. 


\section{References:}

1. Lip GY, Lowe GD, Rumley A, Dunn FG. Fibrinogen and fibrin D-dimer levels in paroxysmal atrial fibrillation: evidence for intermediate elevated levels of intravascular thrombogenesis. Am Heart J. 1996; 131:724-730.

2. Mitusch R, Siemens HJ, Garbe M, Wagner T, Sheikhzadeh A, Diederich KW. Detection of a hypercoagulable state in nonvalvular atrial fibrillation and the effect of anticoagulant therapy. Thromb Haemost. 1996; 75:219223.

3. Ay H, Arsava EM, Tokgozoglu SL, Ozer N, Saribas O. Hyperchomocysteinemia is associated with the presence of left atrial thrombus in patients with nonvalvular atrial fibrillation. Stroke 2003; 34:909-912.

4. Feng D, Edwards WD, Oh JK et al. Intracardiac thrombosis and embolism in patients with cardiac amyloidosis. Circulation 2007; 116:2420-2426.

5. Buyuktas E, Eskazan AE, Borekci S et al. Hypereosinophilic syndrome associated with simultaneous intracardiac thrombi, cerebral thromboembolism and pulmonary embolism. Intern Med 2012; 51:309-313.

6. Cianciulli TF, Saccheri MC, Redruello HJ et al. Right atrial thrombus mimicking myxoma with pulmonary embolism. Tex Heart Inst J 2008; 35(4):454-457.

7. Hammani R, Abid L, Frikha F et al. Intracardiac thrombus in a young man: Do not forget Bechet's disease. Intern Med 2012; 51:1865-1867.

8. Ahmer A, Al-Mondhiry H, Milling TJ Jr., Campbell D. Heparin -induced thrombocytopenia associated with massive intracardiac thrombosis: a case report. Case Report Hematol. 2012; 2012:257023. Epub 2012 Mar 29.

9. Al-Halaseh W, Shah NA, DeGregorio J. Right ventricular thrombus mimicking a myxoma in a 19-year old man. Hospital Physician August 2005; 39-42.

10. Sheikh AY, Schrepfer S, Stein W et al. Right atrial mass after primary repair of an atrial septal defect: thrombus masquerading as a myxoma. Ann Thorac Surg 2007; 84:1742-1744 